Ann. Biol. anim. Bioch. Biophys., I970, 10 (3), 443-457.

\title{
EFFET DU TRAITEMENT THERMIQUE DU TOURTEAU DE SOJA SUR LA VARIATION DE L'ÉQUIPEMENT ENZYMATIQUE DU PANCRÉAS EXOCRINE ET LES PERFORMANCES PONDÉRALES DU RAT APRES SEVRAGE
}

\author{
T. CORRING et A. AUMAITRE \\ avec la collaboration technique de Anne-Marie Gueugneau, Jany Peiniau, \\ et M. LECOURTIER \\ Station de Recherches sur l'Élevage des Porcs, \\ Centre national de Recherches zootechniques, 78 - Jouy-en-Josas \\ Institut national de la Recherche agronomique
}

\section{RÉSUMÉ}

Les phénomènes physiologiques et biochimiques d'adaptation à l'ingestion d'un régime à base de tourtéau de soja cru et cuit ont été étudiés chez le Rat après sevrage.

Un effet défavorable du tourteau de soja cru sur la croissance corporelle des animaux est mis en évidence, et le pancréas subit une hypertrophie. Par ailleurs, les efficacités alimentaire et protidique de cet aliment sont moins élevées que celles du tourteau de soja cuit.

Enfin, l'équipement enzymatique exocrine du pancréas varie après l'ingestion du tourteau de soja cru. Ainsi la biosynthèse de la lipase et de l'amylase semble être inhibée, tandis que celle des enzymes protéolytiques et plus précisément de la chymotrypsine serait stimulée. Cette dernière enzyme se révèle être d'une grande sensibilité à la variation de la nature du régime et montre un pouvoir d'adaptation très élevé au tourtcau de soja cru.

\section{IN'TRODUCTION}

Dès IgI7, Osborne et Mender, avaient constaté que la graine de soja cru, utilisée comme principale source de protéines dans l'alimentation animale, entraînait une croissance médiocre.

Depuis, de nombreuses recherches ont été entreprises dans le but de déterminer les divers effets physiopathologiques de l'ingestion du tourteau de soja cru, en parti- 
culier sur le fonctionnement du pancréas exocrine. De l'ensemble des travaux récents (LyMAN, I957; LYMAN et LEPKOVSKY, I957 ; RACKIS, I965; NitSAN et ALUMOT, I965 ; Combs et al., I967; Gorill et Thomas, I967 ; KoniJn et GugGenheIm, I967 ; MA'AyANI et KuLKA, I968), et quelle que soit l'espèce animale tutilisée, il ressort plusieurs phénomènes :

a) la croissance et la consommation de l'animal sont diminuées,

b) la glande pancréatique subit une hypertrophie,

c) la sécrétion des enzymes pancréatiques en général semble stimulée et la synthèse spécifique des précurseurs protéolytiques paraît affectée.

Cependant, malgré le nombre des résultats obtenus, l'effet de l'ingestion de tourteau de soja cru, à la fois sur les performances de croissance et les phénomènes de la sécrétion pancréatique, reste toujours inexpliqué. Trop fragmentaires, les résultats ne donnent en général qu'un "cliché de la situation pancréatique " à un instant donné, après une adaptation plus ou moins longue de l'animal à son régime. De plus, la réponse enzymatique du pancréas à l'ingestion de tourteau de soja cru, à travers ces résultats, ne met pas en évidence une variation particulière ou bien même une adaptation d'une enzyme à cet aliment.

Dans ce travail, nous avons cherché à étudier l'évolution de l'équipement enzymatique pancréatique et notamment de deux principaux précurseurs protéolytiques, en réponse à l'ingestion de tourteau de soja cru et cuit, chez le Rat après sevrage, pendant une longue période.

\section{MATÉRIEI, E'T MÉTHODES}

\section{Animaux}

Cent Rats mâles de souche Wistar C. F. placés en cage individuelle et pesant en moyenne, en début d'expérimentation, $75 \mathrm{~g}$ à 30 jours d'âge ont été utilisés pour cette étude. Au cours d'une période préexpérimentale de 8 jours, ils ont été nourris d'un régime standard suivant (en p. 1oo) : caséine actique : 12 ; saccharose : 36 ; amidon de maïs : 36,7 ; cellulose Colmacel FI : 2 ; mélange minéral : 4 ; mélange vitaminique : (huile + Vitadone) $: 8 ;$ cystine 0,3 .

a) Tourteau de soja.

\section{Régimes}

Le tourteau de soja cru (1) provenait d'un échantillon délipidé aux solvants chauffé à une température maximale de $55^{\circ}-60^{\circ} \mathrm{C}$. Une partie a été cuite au laboratoire par autoclavage ̀̀ $105^{\circ} \mathrm{C}$ et sous $\mathrm{I}, 2 \mathrm{~kg}$ de pression pendant 45 minutes.

Les tests de cuisson pratiqués sur les échantillons (Delort-Laval et Zelter, 1960 ; OloMUCKi et BoRNSTEIN, rg6o) ont confirmé l'état des tourteaux cru et cuit.

La composition globale du tourteau de soja utilisé est la suivante : p. Ioo

— Humidité................... II,4

- Matières grasses ..............,

- Matières protéiques totales $(\mathbf{N} \times 6,25) \ldots 49,0$

- Protéines solubles dans l'eau...........56,4\% des protéines totales 


\section{b) Composition des régimes.}

Le tableau I rapporte la composition des régimes à base de tourteau de soja (50 p. Ioo en poids). La teneur en protéines brutes de la ration (calculée à $24,5 \mathrm{p}$. Ioo) est largement supérieure aux hesoins protidiques des animaux sans tenir compte de l'équilibre en acides aminés du régime.

Les animaux sont alimentés à volonté, les régimes sont distribués en farine.

\section{TABLEAU I}

Composition des régimes

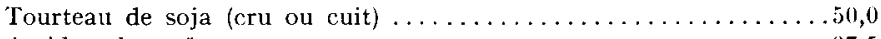

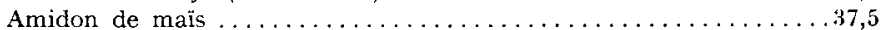

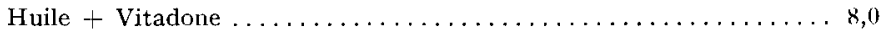

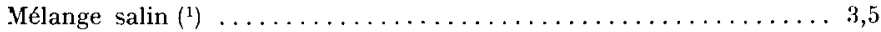

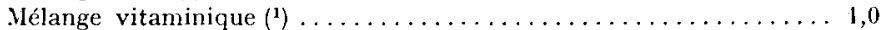

(1) Selon Rírat el al., 1964.

\section{3. Échantillonnage des animaux pendant l'expérimentation}

Deux lots homogènes de 50 rats sont constitués en début d'expérimentation en fonction du poids et du gain de poids acquis durant la période pré-expérimentale. Un lot reçoit le régime à base de tourteau de soja cru, l'autre lot reçoit le régime à base de tourteau de soja cuit. A l'intérieur de chaque lot sont constitués to groupes homogènes d'animaux.

Les différents stades auxquels sont pratiqués les dosages sont les suivants à partir du début de la distribution des régimes expérimentaux : $0,2,3,4,9,15,17,22,29$ et $37^{\mathrm{e}}$ jour.

A chaque stade 5 animaux par régime sont abattus. Les rats sont préalablement mis à jeun I5 heures avant l'abattage.

Tous les animaux sont pesés et la consommation contrôlée individuellement à chaque stade d'étude avant la mise à jeun. Les valeurs moyennes des critères étudiés portent de ce fait sur un nombre de plus en plus réduit d'animaux.

\section{Méthodes et dosages}

Chaque animal est décapité et le pancréas est immédiatement prélevé, débarrassé des tissus gras, pesé et homogénéisé dans l'eau distillée glacée à l'aide d'un Ultra-Turrax (Staufen, type 18/2), permettant de détruire les structures cellulaires. Le contenu intestinal de la partie grêle est recueilli par lavage à l'eau distillée glacée sous légère pression sans extraction du mucus. Tous les contenus intestinaux sont ensuite ajustés au même volume avec de l'eau distillée glacée.

Après homogénéisation du pancréas, il est procédé à la mesure de la teneur du tissu en protéines totales selon la méthode colorimétrique de Folıs. Les activités de la chymotrypsine et de la trypsine du pancréas sont déterminées spécifiquement, après activation des précurseurs par la trypsine cristallisée (Sigma $2 \times$ ) suivant la méthode décrite par ReBoud et al. (I962), à l'aide d'un titrimètre à pH constant (FIgarella et al, 1965). Les mêmes activités enzymatiques sont déterminées dans les contenus intestinaux dans lesquels nous avons vérifié que l'activation n'est pas nécessaire, ainsi que l'avaient montré Figarella et al (1965). Les substrats synthétiques utilisés sont 1'Acétyl-l-Tyrosine-Ethyl-Ester (ATEE) et le Benzoyl-1-Arginine-Éthyl-Ester (BAEE) respectivement pour la chymotrypsine et la trypsine. L'activité est exprimée en $\mu$ moles d'ATEE et de BAEE hydrolysés par minute.

L'activité de la lipase est déterminée dans le pancréas et les contenus intestinaux à l'aide d'un titrimètre à $\mathrm{pH}$ constant par la mesure de la vitesse d'hydrolyse à $37^{\circ} \mathrm{C}$ d'une huile d'olive standard émulsifiée dans la gomme arabique à ro p. Ioo selon la méthode de Desnuelle et al (r962).

L'activité est exprimée en $\mu$ moles d'acides gras libérés par minute.

L'activité de l' $\alpha$ amylase est mesurée dans le pancréas et les contenus intestinaux par la détermination des quantités de sucres réducteurs libérés après une hydrolyse de 20 minutes d'un amidon soluble (PROLABO $2 \mathrm{I}-\mathrm{I}_{53} \mathrm{n}^{\circ} \mathrm{du}$ lot 69 o 17 ) (nombre d'extrémités réductrices libérées).

Les valeurs de ces activités sont exprimées en unités totales pour l'organe, ou sous forme de l'activité spécifique par gramme de tissu ou par mg de protéines tissulaires totales. 


\section{RÉSULTATS}

\section{I. - Croissance et consommation}

Les résultats concernant la croissance des animaux et les quantités d'aliment consommées sont rapportés dans le tableaul 2 et la figure I.

Une diminution de près de $18 \mathrm{p}$. Ioo des performances de croissance se manifeste dès les premiers jours de l'expérimentation chez les animaux soumis au régime à base de tourteau de soja cru. Cette différence par rapport au régime à base de tourteau de soja cuit se maintient jusqu'au dernier stade étudié. Par ailleurs, les écarts notés entre les performances pondérales moyennes (poids et gain de poids) sont significativement différents entre les deux lots et à chacun des stades considérés.

En ce qui concerne la consommation, la quantité totale d'aliment ingéré par les rats nourris du régime à base de tourteau de soja cru, est inférieure dès le premier jour de l'expérimentation jusqu'aux environs du vingtième jour où elle devient égale à la quantité d'aliment ingéré par les rats soumis au tourteau de soja cuit.

Ensuite elle se maintient à une valeur supérieure au lot témoin jusqu'en fin d'expérimentation, si bien que pour l'ensemble de la période les quantités totales sont équivalentes.

\section{Efficacités alimentaire et protidique des régimes.}

Nous avons rapporté dans le tableau 2 et la figure 2 les valeurs du coefficient d'efficacité alimentaire et du coefficient d'efficacité protidique. L'examen de ces données et des courbes correspondantes montre que le régime à base de tourteau de soja cuit présente une meilleure efficacité pour les animaux. En effet, avec ce dernier régime et à chacun des stades considérés, les coefficients d'efficacité alimentaire et protidique sont meilleurs que pour le régime à base de tourteau de soja cru.

\section{2. - Développement de la glande pancréatique}

Les valeurs rapportées au tableau 3 montrent que le poids total du pancréas et la quantité de protéines totales du tissu pancréatique augmentent régulièrement en valeur absolue en fonction du temps. Mais ces valeurs qui sont supérieures dès les premiers jours de l'expérimentation chez les animaux consommant le tourteau de soja cru, traduisent une hypertrophie de la glande pancréatique. Les écarts notés dans le développement du pancréas entre les animaux soumis aux deux régimes sont significativement différents.

Lorsque l'on rapporte ces mêmes résultats soit au poids vif, pour le développement pondéral de la glande, soit au poids frais du pancréas, pour les protéines totales, et cela afin d'éliminer l'effet du développement global de l'animal avec l'âge, les phénomènes observés demeurent identiques (fig. 3 et fig. 4). 
TABLEAU 2

Croissance moyenne des animaux $\left(^{1}\right)$

Consommation d'aliment et efficacités alimentaire et protidique

Régime

Gain de poids total en g/animal

Consommation totale en g/animal ..............

Efficacité alimentaire moyenne

gain de poids/consommation totale .........

Efficacité protidique moyenne

gain de poids/consommation protéines
Soja cuit

$189,6 \pm 1,5$

$118 \pm 14$

$0,312 \pm 0,09$

$1,26 \pm 0,07$
Soja cru

$169,9 \pm 1,5 * *$

$631+12$

$0,270 \pm 0,01 * *$

$1,09 \pm 0,04 * *$

** $\mathrm{P}<0,01$.

\pm Écart-type de la moyenne.

(1) Nombre d'animaux par régime : 50 en début d'expérience; 5 en fin d'expérience.

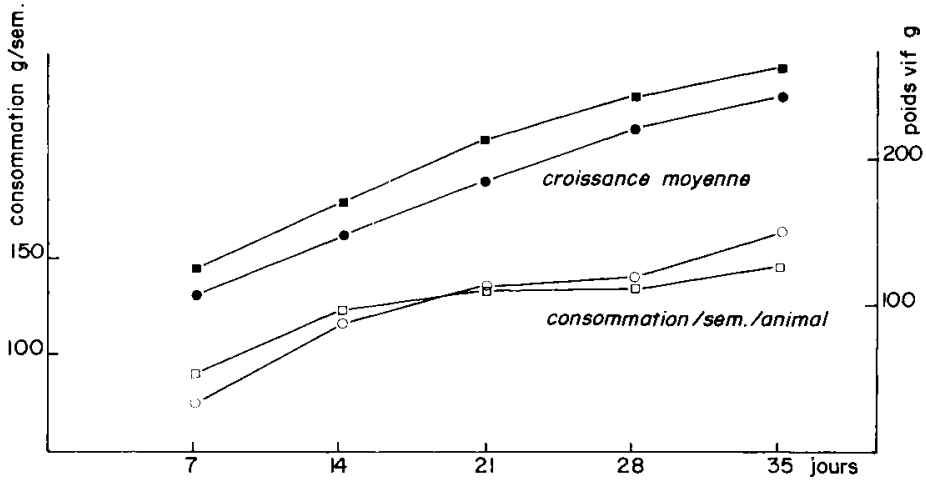

FIG. I. - Croissance et consommation moyennes des animaux nourris du tourteau de soja cuit et cru

- $\square$ Tourteau de soja cuit
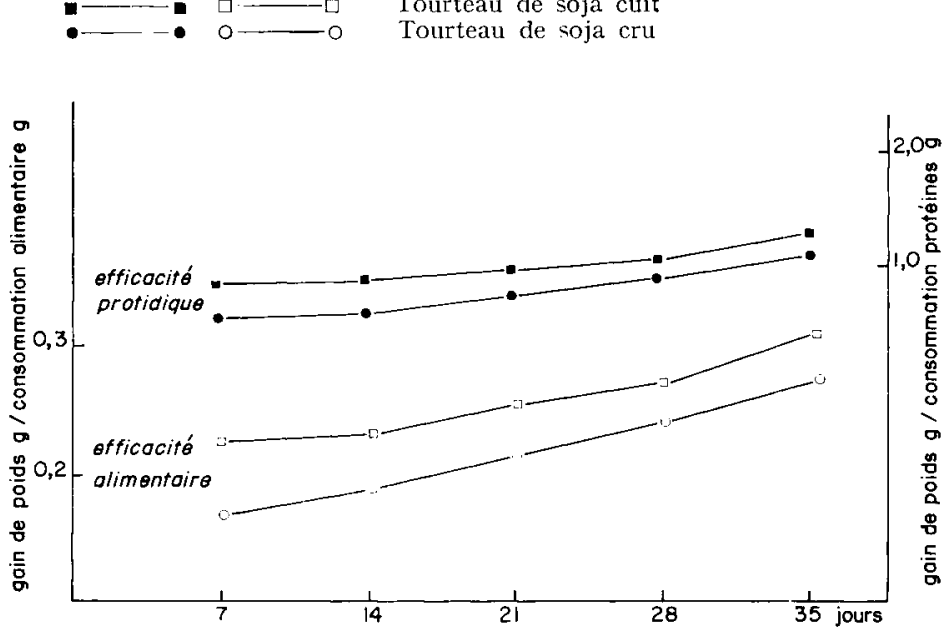

FIG. 2. - Evolution des coefficients d'efficacité alimentaire et protidique chez les animaux notrris du tourteau de soja cuit et cru

$\begin{array}{ll}\text { - } & \text { Tourteau de soja cuit } \\ 0 \ldots \ldots- & \text { Tourteau de soja cru }\end{array}$ 


\section{TABLEAU 3}

Développement pondéral du pancréas et quantité totale de protéines du tissu pancréatique en fonction du temps chez les animaux soumis au tourteau de soja cuit et cru

\begin{tabular}{|c|c|c|}
\hline Régime & Soja cuit & Soja cru \\
\hline \multicolumn{3}{|l|}{$\begin{array}{l}\text { Poids total pancréas frais en } \\
\text { mg : }\end{array}$} \\
\hline 3 jours $\ldots \ldots \ldots \ldots \ldots$ & $508 \pm 82$ & $536 \pm 66$ \\
\hline 15 jours $\ldots \ldots \ldots \ldots \ldots$ & $761 \pm 119$ & $1213 \pm 118$ \\
\hline 37 jours $\ldots \ldots \ldots \ldots \ldots$ & $978 \pm 133$ & $1662 \pm 94$ \\
\hline \multicolumn{3}{|l|}{$\begin{array}{l}\text { Protéines totales du tissu pan- } \\
\text { créatique en mg: }\end{array}$} \\
\hline 3 jours $\ldots \ldots \ldots \ldots \ldots$ & $96 \pm 9$ & $109 \pm 10$ \\
\hline 15 jours $\ldots \ldots \ldots \ldots \ldots \ldots$ & $150 \pm 28$ & $26 ; \pm 35$ \\
\hline 37 jours $\ldots \ldots \ldots \ldots \ldots$ & $18: \pm 23$ & $414 \pm 37$ \\
\hline
\end{tabular}

土 Écart-type de la moyenne.

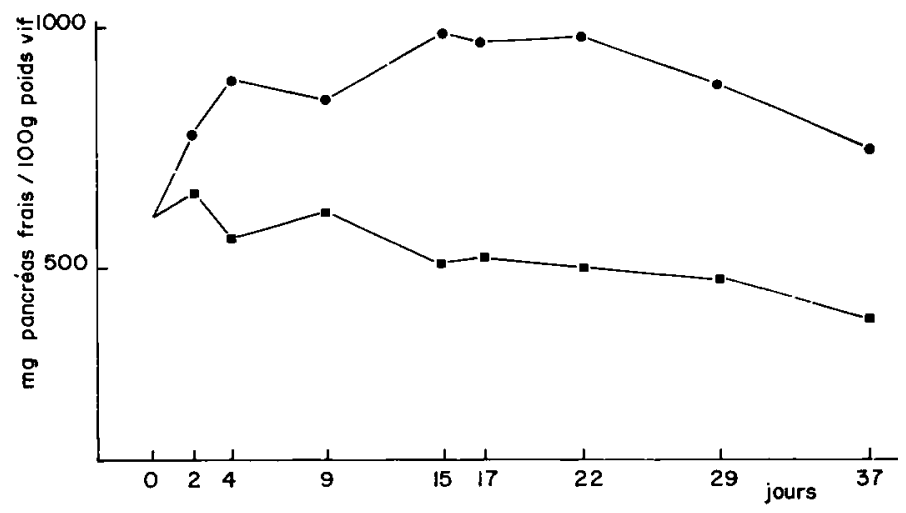

FIG. 3. - Développement pondéral relatif du pancréas des animaux nourris du tourteau de soja cuit et cru

- Tourteau de soja cuit Tourteau de soja cru 


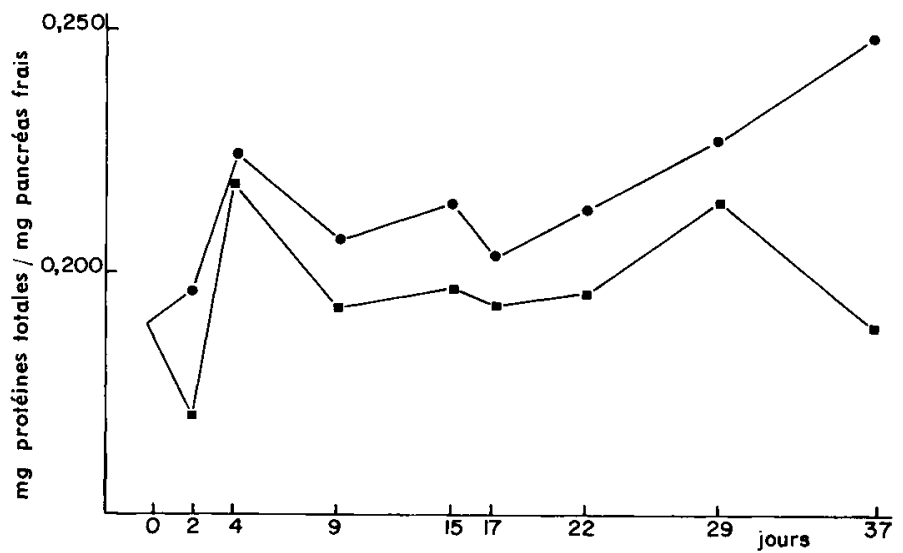

FIG. 4. - Évolution de la quantité de protéines totales dans le pancréas des animaux nourris du tourteau de soja cuit et cru

- Tourteau de soja cuit Tourteau de soja cru

\section{3. - Activités enzymatiques du pancréas}

a) Activités totales.

Les résultats permettent de souligner une différence d'évolution entre les activités protéolytiques d'une part et celles de la lipase et de l'amylase d'autre part (tabl. 4 et 5 ).

\section{TABLEAU 4}

Activités totales de la chymotrypsine et de la trypsine dans le pancréas des rats soumis au tourteau de soja cuit et cru, en fonction de la durée d'expérimentation

\begin{tabular}{|c|c|c|c|c|}
\hline \multirow{2}{*}{$\begin{array}{l}\text { Durée } \\
\text { (jours) }\end{array}$} & \multicolumn{2}{|c|}{ Chymotrypsine ( $\mu$ moles ATEE/mn.) } & \multicolumn{2}{|c|}{ Trypsine $(\mu$ moles BAEE/mn) } \\
\hline & Soja cuit & Soja cru & Soja cuit & Soja cru \\
\hline 0 & $1156 \pm 44$ & $1156 \pm \quad 44$ & $997 \pm 24$ & $997 \pm 24$ \\
\hline 2 & $2103 \pm 117$ & $2598 \pm 293$ & $1112 \pm 47$ & $1165 \pm .62$ \\
\hline 3 & $2247 \pm 61$ & $2817 \pm 111^{* *}$ & $1106 \pm 24$ & $1151 \pm 26$ \\
\hline 4 & $2168 \pm 214$ & $3326 \pm 447 *$ & $1019 \pm 37$ & $1126 \pm 38$ \\
\hline 9 & $2786 \pm 156$ & $4903 \pm 295^{* *}$ & $980 \pm 22$ & $1050 \pm 32$ \\
\hline 15 & $2240 \pm 176$ & $6976 \pm 585^{* *}$ & $1084 \pm 45$ & $1552 \pm 53 * *$ \\
\hline 17 & $2409 \pm 163$ & $7012 \pm 619^{* *}$ & $1080 \pm 35$ & $1632 \pm \quad 4 / 4 * *$ \\
\hline 22 & $2628 \pm 118$ & $8564 \pm 791^{* *}$ & $1050 \pm 18$ & $1796 \pm 120 * *$ \\
\hline 29 & $3636 \pm 269$ & $9400 \pm 769 * *$ & $1152 \pm 26$ & $1908 \pm 74 * *$ \\
\hline 37 & $2762 \pm 162$ & $8456 \pm 778^{* *}$ & $1108 \pm 55$ & $1976 \pm 61 * *$ \\
\hline $\begin{array}{l}* \mathrm{P} \\
* * \mathrm{P}\end{array}$ & & & & \\
\hline
\end{tabular}

L'effet du tourteau de soja cru semble très peu important, voir même nul sur l'activité pancréatique totale de la lipase et de l'amylase. Par contre, les niveaux du chymotrypsinogène et du trypsinogène sont nettement supérieurs dans le pancréas des animaux ingérant cet aliment. 
En ce qui concerne les enzymes protéolytiques, il faut noter la réponse différentielle et, semble-t-il, la plus grande sensibilité de la chymotrypsine. Ainsi, l'activité de cette enzyme montre, en réponse à l'ingestion du tourteau de soja cru, une augmentation par rapport aux valeurs trouvées chez les animaux témoins, dès les premiers jours de l'expérimentation, qui devient de plus en plus importante avec le temps (différence significative). Cela se retrouve en exprimant le rapport des activités chymotrypsine/trypsine pour les deux régimes. Ce rapport augmente régulièrement chez les animaux soumis au tourteau de soja cru et passe de 2 à 4 du premier au neuvième jour alors que, pendant la même période, il ne varie pas chez les animaux témoins. La trypsine réagit également mais seulement à partir $\mathrm{du}$ quinzième jour, et l'augmentation de son activité est légère puisque en fin d'expérimentation, le rapport des activités trypsiques pour les 2 régimes est de $\mathrm{I}, 2$ alors que celui des activités chymotrypsiques est de 3,4 .

\section{TABLEAU 5}

Activités totales de la lipase et de l'amylase dans le pancréas des rats soumis au tourteau de soja cuit et cru, en fonction de la durée d'expérimentation.

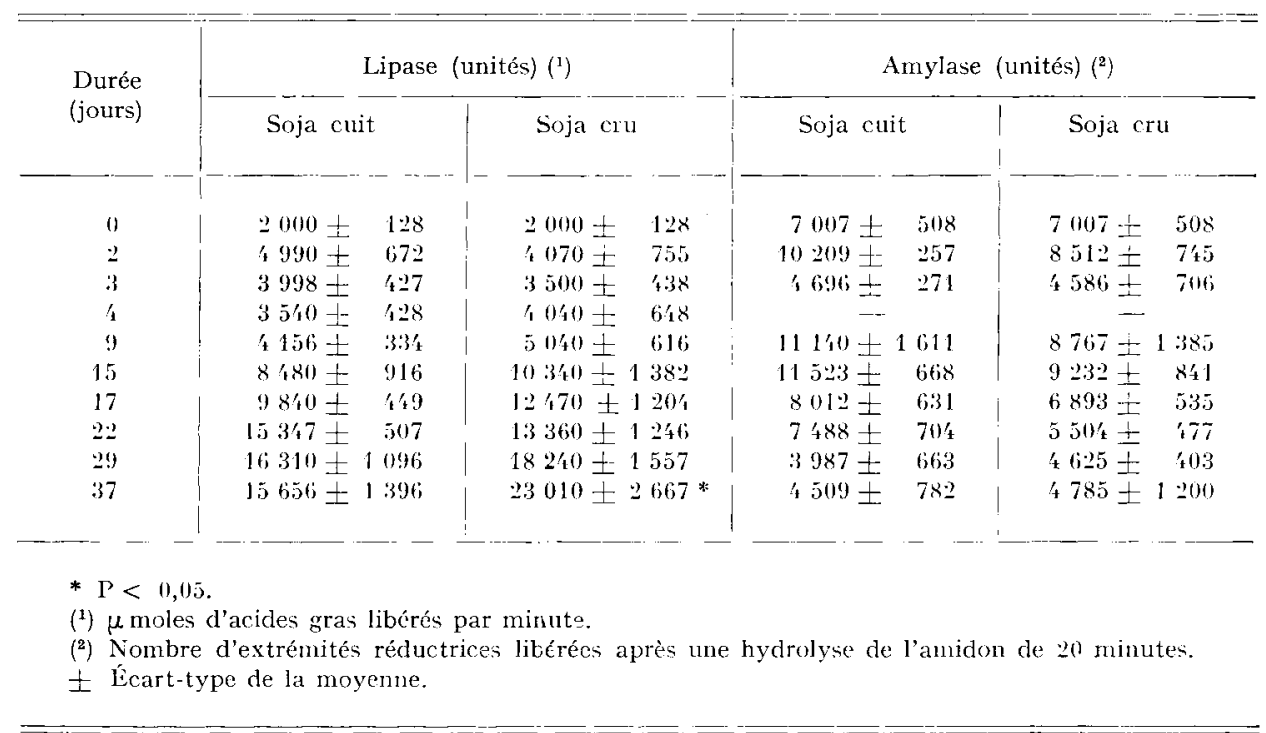

Enfin, il faut souligner que l'écart-type des activités moyennes rapportées au tableau 4 varie peu, ce qui prouve l'homogénéité des résultats. Il n'en est pas de même lorsque l'on considère les valeurs rapportées au tableau 5 concernant les activités totales de la lipase et de l'amylase; l'écart-type est ici plus important, ce qui ne nous a pas permis de mettre en évidence des différences significatives entre les valeurs déterminées sauf pour certains stades.

b) Activités spécifiques.

A partir des activités enzymatiques rapportées au poids frais de l'organe (fig. 5 , 6 et 7) ou aux protéines totales du tissu pancréatique, la différence notée précédem- 


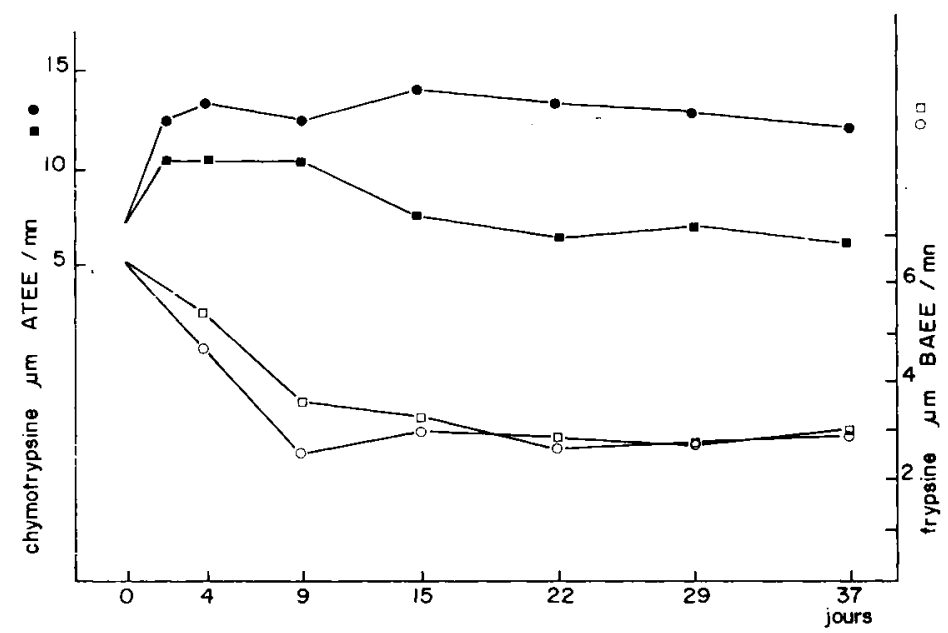

FIG. 5. - Activités enzymatiques de la chymotrypsine et de la trypsine par mg de pancréas frais dans le pancréas des animaux nourris du tourteau de soja cuit et cru
Chymotrypsine
$\{$ Tourteau de soja cuit
Trypsine
Tourteau de soja cru
Tourteau de soja cuit
$\{$ Tourteau de soja cru

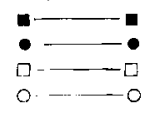

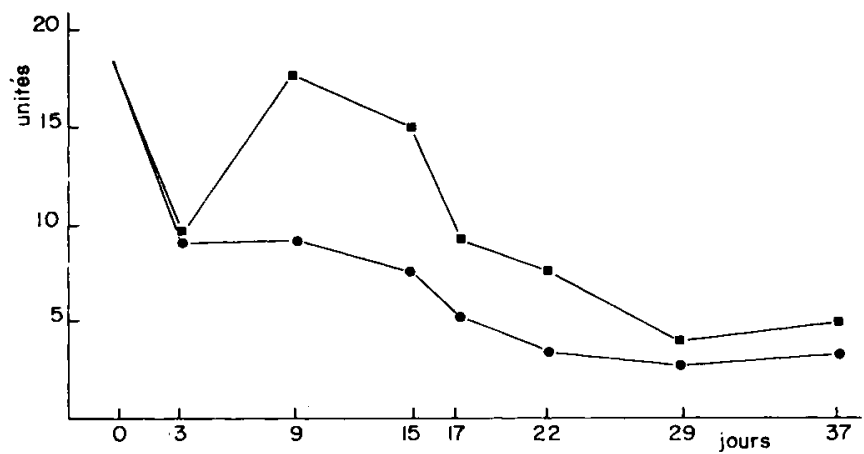

FIG. 6. - Activité enzymatique de l'amylase par ma de pancréas frais dans le pancréas des animanx nourris du tourtean de soja cuit et cru - Tourteau de soja cuit
Tourteau de soja cru

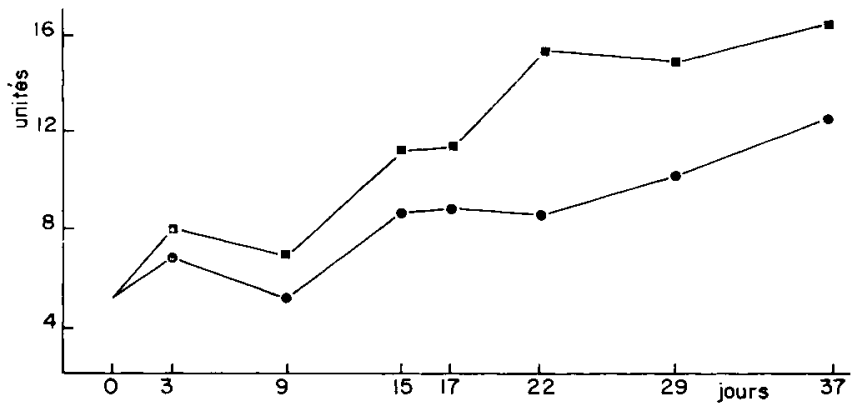

FIG. 7. - Activitê enzymatique de la lipase par mg de pancréas frais dans le pancréas des animaux nourris du tourteau de soja cuit et cru Tourteau de soja cuit 
ment entre les enzymes protéolytiques d'une part et la lipase et l'amylase d'autre part est plus nettement mise en évidence.

De plus, on observe que le niveau du chymotrypsinogène pancréatique est significativement supérieur dès les premiers jours qui suivent l'ingestion du tourteau

\section{TABI,EAU 6}

Activités totales chymotrypsique et trypsique dans les contenus intestinaux des animaux nourris du tourteau de soja cuit et cru, en fonction de la durée d'expérimentation

\begin{tabular}{|c|c|c|c|c|}
\hline \multirow{2}{*}{ Durée } & \multicolumn{2}{|c|}{$\begin{array}{c}\text { Chymotrypsine } \\
(\mu \text { moles ATEE } / \mathrm{mn})\end{array}$} & \multicolumn{2}{|c|}{$\begin{array}{c}\text { Trypsine } \\
(\mu \text { molesBAEE } / \mathrm{mn})\end{array}$} \\
\hline & Soja cuit & Soja cru & Soja cuit & Soja cru \\
\hline $15^{\mathrm{e}}$ jour & $132 \pm 20$ & 153 次 38 & $41 \pm 4$ & $33 \pm$ \\
\hline $17^{\mathrm{e}}$ jour $\ldots$ & $217 \pm 33$ & 230 起 35 & $48 \pm 8$ & $45 \pm$ \\
\hline $22^{\mathrm{e}}$ jour $\ldots$ & $183 \pm 20$ & $170+32$ & $32 \pm 2$ & $30 \pm 4$ \\
\hline 29 e jour $\ldots \ldots$ & $203 \pm 2$ & $202 \pm 32$ & $38 \pm 6$ & $62 \pm 17$ \\
\hline $37^{\mathrm{e}}$ jour $\ldots \ldots$ & $194 \pm 38$ & $177 \pm 9$ & $51 \pm 5$ & 't't 2 \\
\hline
\end{tabular}

\pm Écart-type à la moyenne.

\section{TABLEAU 7}

Activités totales lipasique et amylasique dans les contenus intestinaux des animaux nourris du tourteau de soja cuit et cru, en fonction de la durée d'expérimentation

\begin{tabular}{|c|c|c|c|c|c|}
\hline & & \multicolumn{2}{|c|}{ Lipase (unités) (') } & \multicolumn{2}{|c|}{ Amylase (unités) (²) } \\
\hline & & Soja cuit & Soja cru & Soja cuit & Soja cru \\
\hline $15^{\mathrm{e}}$ jour & $\ldots \ldots \ldots$ & $81 \pm 28$ & $24 \pm 4$ & $247 \pm$ & $157 \pm 12$ \\
\hline $17^{\mathrm{e}}$ jour & $\ldots \ldots$ & $110 \pm 19$ & $40 \pm 5$ & $95 \pm 20$ & $59 \pm 9$ \\
\hline $22 \mathrm{e}$ jour & $\ldots \ldots$ & $10^{\prime} \pm 11$ & $39 \pm 5$ & $4: 1 \pm 100$ & $76 \pm 17$ \\
\hline $29^{\mathrm{e}}$ jour & $\ldots \ldots \ldots$ & $16 \div \pm 12$ & $90 \pm 23$ & $1298 \pm 300$ & $90 \pm 13$ \\
\hline $37^{e}$ jour & $\ldots \ldots \ldots$ & $210 \pm 37$ & $80 \pm 14$ & $651 \pm \quad 6 \prime$ & $4: 25 \pm 80$ \\
\hline
\end{tabular}

(') 4 moles d'acides gras libérés par ninute.

(2) Nombre d'extrémités réductrices libérées après une hydrolyse de l'amidon de 20 minutes.

土 : écart-type de la moyenne.

de soja cru, alors que celui du trypsinogène est identique pour les deux régimes. Par ailleurs, les activités de la lipase et de l'amylase sont plus importantes dans le pancréas des animaux alimentés à partir du tourteau de soja cuit.

\section{4. - Activités enzymatiques dans les contenus intestinaux}

Les valeurs des activités enzymatiques totales dans les contenus intestinaux montrent une variabilité importante. Cependant, quel que soit le régime ingéré, les 
quantités d'enzymes protéolytiques sont identiques à chacun des stades de 1'expérimentation, alors que les activités lipasique et amylasique sont significativement plus faibles dans les contenus intestinaux des animaux nourris du régime à base de tourteau de soja cru, mise à part une valeur aberrante pour l'amylase (tab1. 6 et 7 ).

\section{DISCUSSION}

\section{Croissance des animaux et consommation d'aliment}

Le Rat récemment sevré répond à un traitement thermique du tourteau de soja incorporé dans sa ration. Ainsi, pour l'ensemble de la période considérée, la croissance est supérieure de I 2 p. roo en moyenne avec le tourteau de soja cuit par rapport au tourteau de soja cru et le phénomène s'observe tout au long de l'expérimentation. Cet effet dépressif du tourteau de soja cru sur la croissance des animaux à différents stades de leur vie a été noté par ailleurs sur le Rat par Lyman (I957), Lyman et LEPKOvSKy (I957), KoniJN et GUGGenHeIm (I967); sur le Porcelet par Hooks et al. (I965), Sheppard et al (I967) ; sur le Poulet par Pubols et al. (I964), Nitsan et al. (Ig65).

L'évolution de la quantité relative d'aliment consommé est intéressante à considérer. La consommation de tourteau de soja cru est d'abord plus faible que calle de l'aliment témoin, puis après 20 jours d'expérimentation, elle devient supérieure. A notre connaissance, aucun résultat de ce type n'a été déjà rapporté. En fin d'expérimentation, les quantités totales d'aliment ingéré sont sensiblement identiques. On peut penser qu'il existe une adaptation de l'animal avec le temps au tourteau de soja cru, car après un effet dépressif sur la consommation, on observe une stimulation de celle-ci.

Néanmoins et malgré cette observation, on constate que l'efficacité globale de l'aliment est plus faible lorsqu'il contient du tourteau cru. Ainsi les efficacités alimentaire et protidique sont inférieures de I7 p. Ioo en moyenne au tourteau cuit, et cela tout au long de l'expérimentation. Ces résultats confirment les données de HaINes et Lyman (I96I), de BorChers (I96I) et de Rackis (I965). De plus, on peut penser, d'après les̃ observations de Combs et al. (I967) sur le Porcelet; de LEPKOvSKY et al. (I965), Aso et al. (I967) sur le poulet, à un effet favorable du traitement thermique du tourteau de soja sur la digestibilité des protéines du régime. Une telle hypothèse peut également être étayée par les résultats de Hooks et al. (I965) sur le Porcelet et de BARNEs et Kwong (I965) sur le Rat qui ont montré que la cuisson du tourteau de soja améliore la disponibilité des acides aminés de la ration. Cependant, il importe de rappeler que dans cette expérience les régimes ont été calculés de telle sorte que les besoins en acides aminés soient largement couverts. Pour le taux élevé de protéines utilisé dans l'expérience, les efficacités médiocres ne peuvent s'expliquer que par une très forte diminution de la digestibilité sous l'influence du tourteau de soja cru.

Enfin, notons que l'augmentation des efficacités protidique et alimentaire souligne cependant les phénomènes d'adaptation avec l'âge de l'animal au régime, quel que soit le traitement appliqué au tourteau. Mais l'écart entre les deux régimes demeure ainsi que l'ont observé Saxena et al. (I963) chez le Poulet. 


\section{Développement de la glande pancréatique}

Nos résultats indiquent un développement pondéral relatif plus important du pancréas des animaux recevant le tourteau de soja cru (40 p. Ioo en plus), ce qui se traduit également par une augmentation de la quantité relative de protéines tissulaires (Io p. Ioo en plus). Ces résultats sont classiques chez le Rat. (L y MAN, I957; KonIjN et GuGGENHEIm, I967) et sont attribués à une hypertrophie des cellules plutôt qu'à un développement par hyperplasie.

Cependant il existe un comportement différent du Rat et du Poulet, car le phénomène d'hypertrophie du pancréas s'observe à tous les âges considérés chez le Rat, alors qu'il s'atténue avec l'âge chez le Poulet (Nitsan et Alumot, I965; SAXENA et al, I963). On peut done penser que l'effet particulier du tourteau de soja cru se manifeste chez le Rat par une augmentation de l'activité cellulaire du pancréas, et notamment par une augmentation de la synthèse protéique, ainsi que l'ont montré BARnEs et KWONG (I965).

\section{Activités enzymatiques exocrines}

L'intense activité physiologique du pancréas exocrine que semblent traduire l'hypertrophie pancréatique et la quantité de protéines totales dans le tissu, chez les animaux soumis au tourteau de soja cru, paraît être confirmée lorsque l'on considère l'évolution des activités enzymatiques étudiées.

Les activités totales par pancréas de la lipase et de l'amylase semblent peu affectées par le traitement thermique du tourteau de soja. Fn effet, on n'observe aucune variation de ces activités, en accord avec les résultats de MA'AYANI et KUL,KA (I968) en ce qui concerne du moins l'amylase.

Ces données doivent cependant être nuancées lorsque l'on exprime les activités spécifiques (par gramme de tissu frais ou par milligramme de protéines totales). On observe ainsi que les activités de la lipase et de l'amylase sont significativement inférieures dans le pancréas des rats soumis au tourteau de soja cru. Ces résultats laisseraient supposer une inhibition de la biosynthèse de ces enzymes.

Par contre, lorsque l'on considère l'effet du tourteau de soja cru sur le comportement des enzymes protéolytiques étuaiiées, les résultats nous permettent de conclure à une augmentation de l'activité totale par pancréas de la chymotrypsine et de la trypsine et cela à tous les stades considérés. Cependant, comme précédemment, ces données doivent être nuancées. L'expression sous forme d'activité totale par glande confond en partie le développement général de l'équipement enzymatique avec celui de l'organe en fonction du temps. Mais l'expression des activités spécifiques permet de mettre en évidence une augmentation de l'activité chymotrypsique seule sous l'effet du tourteau de soja cru, et non de la totalité d'une "activité trypsique " du pancréas comme l'ont signalé CHERNICK et al. (I948) ; LEPKOVSKy et al. (I965) et Ma'ayani et KulKa (I968).

Nous pouvons ici souligner encore le comportement différent du Rat et du Poulet car chez ce dernier c'est la trypsine qui répond à l'ingestion du tourteau de soja cru (Pubois et al. I964). La chymotrypsine nous apparaît donc chez le Rat comme une enzyme susceptible d'une très grande adaptation. De telles propriétés de la chymo- 
trypsine avaient déjà été soulignées par BEN ABDELJLII, et al. (I964), REBOUD et al. (I966) suivant le taux de protéines de la ration chez le Rat, et par SNook et MEYER (I964) chez le Rat et PekAs (I964) chez le Porcelet suivant la nature des protéines ingérées.

Les résultats d'activités enzymatiques protéolytiques déterminées au niveau intestinal nous permettent de conclure que cette augmentation de l'activité spécifique de la chymotrypsine est due à une biosynthèse accrue de cette enzyme au niveau de la glande. En effet, quel que soit le régime, nous obtenons des valeurs identiques de ces activités dans les contenus intestinaux. Pour les animaux mis à jeun depuis I5 heures, l'excrétion enzymatique dans le duodénum est minime et les activités déterminées au niveau de la glande prouvent que son pouvoir de biosynthèse est essentiellement augmenté pour le chymotrypsinogène, sous l'effet du tourteau de soja cru.

De même les activités de la lipase et de l'amylase déterminées dans les contenus intestinaux semblent indiquer que l'hypothèse de l'inhibition de la biosynthèse de ces deux protéines enzymatiques serait la seule à retenir. Ce qui pourrait confirmer indirectement, et en ce qui concerne la lipase, le résultat de NESHEIM $e t$ al. (I962) qui a montré un effet défavorable du tourteau de soja cru sur l'absorption des graisses.

\section{CONCLUSION}

L'ingestion du tourteau de soja cru par le Rat en croissance se traduit par:

a) une dépression de la croissance qui peut s'expliquer au début par une diminution systématique, quel que soit l'âge, des efficacités alimentaire et protidique de l'aliment ;

b) une stimulation du pancréas qui répond par une augmentation de la taille et de la quantité de protéines totales;

c) une stimulation de la biosynthèse enzymatique, protéolytique et notamment du chymotrypsinogène. La biosynthèse de la lipase et de l'amylase, en revanche, est inhibée.

Reçu pour publication en avril 1970

\section{SUMMARY}

EFFECT OF HEAT TREATMENT OF SOYA BEAN MEAI,

ON CHANGES IN THE ENZYME APPARATUS OF THE PANCREAS

AND WEIGHT OF RATS AFTER WEANING

The object of the work was to study changes in the enzyme apparatus of the pancreas, particularly of two proteolytic precursors, after ingestion of raw or cooked soya bean meal, in growing rats during 37 days. The diets had 50 per cent soya bean meal. They were given to appetite to 2 groups of 50 rats. Mean weight of the rats and mean intake were recorded at each sampling.

Activities of chymotrypsin, trypsin, lipase and amylase were estimated immediately after sampling the pancreas. Activity of the proteolytic enzymes were estimated with specific synthetic substrates. The effect of raw soya bean meal was shown in the following way: 
I. There was a depression in growth and a systematic depression in utilization of feed and of protein (table 2 ; fig. 1 and 2 ).

2. There was an incrase in the size of the pancreas and in amount of total pancreatic protein (table 3 ; fig. 3 and 4 ).

3. There was an increase in total activity of chymotrypsin and of trypsin (table 4). Biosynthesis of chymotrypsinogen only was greatly stimulated (fig. 5), while those of lipase and of amylase were inhibited (fig. 6 and 7 ).

The results in general confirm the findings of numerous research workers. However, this study has made it possible to demonstrate the great sensitivity of synthesis of chymotrypsinogen in response to inclusion of raw soya bean meal in the feed.

\section{RÉFÉRENCES BIBLIOGRAPHIQUES}

Aso K., Takahashi Y., Tanaka Y., I967. The influence of soybean trypsin inhibitors on the growth, pancreatic changes and digestibility of diet in chicks. Jap. J. Zootech. Sci., 38, 435-442,

Barnes R. H., Kwong E., 1965. Effect of soybean trypsin inhibitors and penicillin on cystin biosynthesis in the pancreas and its transport as exocrine protein secretion in the intestinal tract of the rat. $J$. Nutr., 86, 245-252.

Ben Abdeljuil A., Desnuelle P., I964. Sur l'adaptation des enzymes exocrines du pancréas à la composition du régime. Biochim. Biophys. Acta, 81, I $36-\mathrm{r} 49$.

Borchers R., I96I. Counteraction of the growth depression of raw soybean oil meal by amino acid supplements in weanling rats. J. Nutr., 75, 330-334.

Chernick S. S., Lepkovsky S., Chaikoff L. L., I948. A dietary factor regulating the enzyme content of the pancreas. Changes induced in size and proteolytic activity of the chick pancreas by the ingestion of raw soybean meal. Amer. J. Physiol., 155, 33 .

Combs G. E., Conell R. G., Berry T. M., Wallace J., 1967. Effect of raw and heated soybeans on gain, nutrient digestibility, plasma amino-acids and other blood constituents of growing swine. J. Anim. Sci., 26, ro67-ro7r.

Delort-Laval J., Zelter S.-Z., ig60. État actuel du problème de la qualité des tourteaux de soja et de son contrôle par des tests chimiques. Ind. alim. Anim., 110, 25-34.

Desnuelle P., Reboud J. P., Ben Abdeljlil A., 1962. Influence of the composition of the diet on the enzyme content of rat pancreas, IN : Ciba Foundation Symposium on the exocrine pancreas, Churchill (Londres) p. 90.

Figarella C., Taulier J., Sarles M., 1965. Dosage de la chymotrypsine et de la trypsine dans le suc duodénal. Bull. Soc. Chim. Biol., 47, 679-686.

Gorill, A. D. L., Thomas J. W., I967. Body weight changes, pancreas size and enzyme activity, and proteolytic enzyme activity and protein digestion in intestinal contents from calves fed soybean and milk protein diets. J. Nutr., 92, $2 \mathrm{I}_{5}-223$.

Haines P. C., Lyman R. L., rg6r. Relationship of pancreatic enzyme secretion to growth inhibition in rats fed soybean trypsin inhibitor. $J$. Nutr., 74, 445-452.

Hooks R. D., Hays V. W., Speer J. C.,Mc CaIL J. T., I965. Effect of raw soybeans on pancreatic enzyme concentrations and performance of pigs. J. Anim. Sci., 24, 894.

KoniJn A. M., Guggenheim K., 1967. Effect of raw soybean flour on the composition of the rat pancreas. Proc. Soc. Exp. Biol. Med., 126, 65-67.

LEPKovSKY S., et al, I965. The effect of raw soja beans upon the digestion of proteins and upon the function of the pancreas of intact chickens and of chickens with ileostomies. Brit. J. Nutr., 19, 4I-56.

Lyman R. L., 1957. The effect of raw soybean meal and trypsin inhibitor diets on the intestinal and pancreatic nitrogen in the Rat. J. Nutr., 62, 285-294.

Lyman R. L., Lepkovsky S., I957. The effect of raw soybean meal and trypsin inhibitor diets on pancreatic enzyme secretion in the rat. J. Nutr., 62, 269-284.

Ma'Ayani S., Kulka R. G., I968. Amylase, procarboxypeptidase and chymotrypsinogen in pancreas of chicks fed raw or heated soybean diet. J. Nutr., 96, 363-367.

Nesheim M. C., Garlich J. D., Hopkins D. T., ig62. Studies on the effect of raw soybean meal on fat absorption in young chicks. J. Nutr., 78, 89-94.

Nitsan Z., Alumot E., Ig65. The effect of raw soybean levels in the diet on the proteolytic activity and pancreatic hypertrophy in the growing chick. Poult. Sci., 44, 1210-1214.

Olomucki E., Bornstein S., 1960. The dye absorption test for the evaluation of soybean meal quality. J. Assoc. Offic. Agr. Chem., 43, 440-442.

Osborne T. B., Mendel L. B., I9I7. The use of soybean as food. J. Biol. Chem., 32, 369.

Pekas J. C., May V. W., Thompson A. M., I964. Exclusion of the exocrine pancreatic secretion : effect on digestibility of soybean and milk protein by baby pigs at various ages. J. Nutr., 82, $277-285$. 
Pubols M. H., Saxena H. S., McGinnis J., 1964. Pancreatic enzyme levels in chicks fed unheated meal. Proc. Soc. Exp. Biol. Med., 117, 713-7I7.

RACKIS J. J., I965. Physiological properties of soybean trypsin inhibitors and their relationship to pancreatic hypertrophy and growth inhibition in rats. Fedn. Proc., 24, r488-I 493.

Reboud J. P., Ben Abdeljill A., Desnuelle P., ig62. Variation de la teneur en enzymes du pancréas de rat en fonction de la composition des régimes. Biochim. Biophys. Acta., 58, 326-337.

Reboud J. P., Marchis-Mouren G., Pasero L., Cozzone A., Desnuelle P., ig66. Adaptation de la vitesse de biosynthèse de l'amylase pancréatique et du chymotrypsinogène à des régimes riches en amidon ou en protéines. Biochim. Biophys. Acta., 117, 351-367.

Rérat A, Février C., Henry Y., Lougnon J., rg64. Évolution de la composition corpor slle chez le rat blanc en croissance. Ann. Biol. anim. Bioch. Biophys., 4, 35-47.

SAXENA H. S., Jensen L. S., McGinnis J., Ig63. Influence of age on utilization of raw soybean meal by chickens. J. Nutr., 80, 391-396.

Sheppard S., May V. W., Speer V. C., Ewans R. C., I967. Effect of antibiotic on response of pigs to raw soybean and solvent soybean meal. J. Anim. Sci., 26, I478.

SNook J. T., Meyer J. H., I964. Response of digestive enzymes to dietary protein. J. Nutr., 82, 409-4I 4 . 